\title{
Hydrodynamic Analogy to Special Relativity
}

\author{
Xue-Nong Chen \\ Institute for Nuclear and Energy Technologies, Karlsruhe Institute of Technology, Karlsruhe, Germany \\ Email:xue-nong.chen@kit.edu
}

How to cite this paper: Chen, X.-N. (2016) Hydrodynamic Analogy to Special Relativity. World Journal of Mechanics, 6, 406418.

http://dx.doi.org/10.4236/wjm.2016.610029

Received: September 19, 2016

Accepted: October 22, 2016

Published: October 25, 2016

Copyright $\odot 2016$ by author and Scientific Research Publishing Inc. This work is licensed under the Creative Commons Attribution International License (CC BY 4.0).

http://creativecommons.org/licenses/by/4.0/

\section{Abstract}

By studying of a slender body moving in a fluid wave-medium, e.g., in air or in shallow water, it was found that the hydrodynamic momentum mass and the total energy of the fluid field can be expressed in forms of $m=m_{o} / \sqrt{1-(v / c)^{2}}$ and $E=m c^{2}$, where $v$ is the body moving speed, $c$ is the wave speed and $m_{0}$ is the hydrodynamic mass at the zero speed. Thus a hydrodynamic analogy to the relativistic particle motion in vacuum can be traced. The velocity dependence of mass and the mass-energy equivalence are universal for any wave medium, which should not be regarded as a consequence of relative Lorentz time-space, but one of the existence of wave in the medium. Its further inference leads to an even more significant physical picture. If the mass particle moves in an unbounded space at a supercritical speed, i.e. $v>c$, waves are generated and radiated from it, like the Mach waves by the supersonic plane, and the particle itself experiences a resistance as reaction from the wave radiation. By an extension of this analogy, it can be interred from a hydrodynamic superconductive phenomenon that particles or waves can move possibly at a superluminal speed without experiencing any resistance through a tunnel (a bounded space) under certain conditions. Therefore the speed of light is not the limit of our physical world and superluminal phenomena are possible.

\section{Keywords}

Hydrodynamic Mass, Water Waves, Special Relativity, Velocity Dependence of Mass, Mass-Energy Equivalence

\section{Introduction}

The problem studied here concerns a slender body moving in a medium, which supports a kind of wave, e.g. shallow water wave or sound wave, characterised by a wave speed $c$. The medium is assumed to be linear and nondispersive. It has been well-known since 1898 [1] that the D'Alembert's paradox holds theoretically for the 
case of subcritical steady motion and the wave speed is a singular point of body moving speed. Physically if a body moves at the wave speed, the momentum and energy produced by the body can not spread out from the body. Contrarily they are accumulated around the body and become infinitely large. Therefore the wave speed is a critical speed for the body motion. In the case of slender body, it was shown in [2] that every integral quantity, e.g. hydrodynamic force or moment, carries a factor $\gamma=1 / \sqrt{1-(v / c)^{2}}$ in subcritical cases.

On the other side, it is always intriguing to think why electromagnetic waves can travel in a vacuum. According to classical understanding of medium waves, there might be a carrier medium for such waves in vacuum. This imagined carrier medium is called aether. One tried to detect an aether but never succeeded in it. Based on this fact Einstein in [3] and [4] developed his special theory of relativity. Its kinematic basis is the Lorentz transformation, which can be derived from two postulates, relativity and constancy of the speed of light, proposed by Einstein. Two famous dynamic consequences are drawn from its kinematics, the velocity dependence of mass and the massenergy equivalence, namely in mathematical formulas

$$
m=m_{o} / \sqrt{1-\beta^{2}} \text { and } E=m c^{2},
$$

where $\beta=v / c, v$ is the relative velocity of the moving body with respect to the observer and $c$ is the speed of light. Since then one needs no more aether concept, but it doesn't mean that the vacuum is not a medium.

This paper deals with following questions. Do moving bodies in classical wave media within the frame of Newtonian mechanics possess also these two dynamic properties? If it is true, what does it mean in physics? What can be inferred from it for the general physics?

The concept of hydrodynamic mass and energy is at first summarized and an associated story about applying it to explain the mass increase of a moving particle is reviewed in retrospect. The reason for the failure of this explanation trial is pointed out. Then the two quantities are calculated in detail for a special case of a slender body and the mathematical similarity to the special relativity is presented. By this classical hydrodynamic example the physical reason for the velocity dependence of mass and the mass-energy equivalence can be clarified, namely it is due to the interaction with wave field (medium). Two further thinking experiments can be carried out. First, what happens, if a mass particle moves at a superluminal speed in a free space? Second, is there any possibility, where particles and waves move at a superluminal speed without decaying? The answers derived from the analogy are amazing. Moreover, in Appendix, the author shows three theorems in the hydrodynamics, which have the same mathematical form as the three basic laws of Coulomb, Ampere and Faraday in electrodynamics correspondingly. This means that the linear wave equation in the hydrodynamics can be set up in the same way as in the electrodynamics, i.e. from the three field observation laws without considering the wave field as a medium, although it is always derived in a different way based on Newtonian continuum mechanics. The most important implication of this analogy is that the velocity dependence of mass and 
the mass-energy equivalence are universal for any wave medium, which should not be regarded as a mathematical consequence of Lorentz transformation, but a physical consequence of the existence of wave in the medium.

\section{Hydrodynamic Mass}

Let us first briefly state the well-known result of a rigid body motion in an incompressible ideal fluid, c.f. [5]. Consider a body moving with a velocity $v$ in a straight line in unbounded fluid. The motion of the rigid body causes motion of fluid around it, thus the fluid has also momentum and kinetic energy, which can be expressed as $m v$ and $\frac{1}{2} m v^{2}$, respectively, where $m$ is added mass or hydrodynamic mass. Newton's three laws hold in the same form as that for the rigid body motion in a free space. The presence of the fluid only effectively increases the mass of the rigid body from $m_{b}$ to $m_{b}+m$. The mass $m_{b}+m$ is called the virtual mass of the body. In other words the virtual mass is obtained by increasing the mass of body $m_{b}$ by the added (hydrodynamic) mass $\mathrm{m}$. It would appear also in hydrodynamic experiments that all moving bodies, in so far as the motion takes place in a fluid medium, should be affected by the added mass.

Generally an incompressible potential flow is governed by Laplace equation,

$$
\nabla^{2} \varphi=0 \text {, }
$$

and the added mass is a tensor of 2 nd order and defined for arbitrary motion of the body through the momentum $\boldsymbol{p}=\left(p_{1}, p_{2}, p_{3}\right)$

$$
p_{i}=\sum_{i} m_{i j} v_{i}
$$

as

$$
m_{i j}:=\int_{V} \rho \frac{\partial \phi_{i}}{\partial x_{j}} \mathrm{~d} V=-\int_{S} \rho \phi_{i} n_{j} \mathrm{~d} S,
$$

where $\phi_{i}$ is unit potential, i.e.

$$
\varphi=\sum_{i} v_{i} \phi_{i}
$$

and $\boldsymbol{n}=\left(n_{1}, n_{2}, n_{3}\right)$ is the body surface outward unit normal vector. The above definition of added mass is generally valid for the hydrodynamic mass in any potential flow case, no matter if the problem is governed by Laplace equation or a wave equation. Newton's second law reads, the net force experienced by the body is equal to the change of momentum per unit time,

$$
F_{i}=\frac{\mathrm{d}}{\mathrm{d} t} \sum_{j}\left(m \delta_{i j}+m_{i j}\right) v_{j}
$$

The added mass reflects the effect of medium on the body motion. In terms of it the body motion is still described by the Newton's second law, where the effective mass is the sum of the mass of solid body and the added mass. If the added mass is taken rightly into account, the question, whether a medium exists, doesn't matter. 
Historically the concept of added mass was so instructive, that a series of investigations had been carried out by Lorentz, Thomson, Abraham and Kaufman, which were contributed to explain the mass increase of a moving particle, e.g. a moving electron, in electromagnetism based an analogy to the added mass, c.f. [6] (p. 155). At the beginning Thomson in [7] suggested and calculated the effective mass is in the form

$$
m=m_{o}+\mu
$$

where $\mu$ could be called the electromagnetic mass similarly to the hydrodynamic mass. But it hardly explained the phenomenon. The final experimental verdict in the years 1914-1916 is in favour of relativity. The reason for this failure can be pointed out. The moving particle interacts with electromagnetic fields and its mass is a result of this interaction, in other words, the mass of a particle is the inertial measure of the moving particle in the wave field. The mass manifestation can not appear without the wave field that is interactive with it. Therefore the hydrodynamic added mass, which is the result of the interaction of moving body and flow field, is not corresponding to the electromagnetic mass but to the whole mass. The increase of mass with velocity is merely due to the wave medium field, as shown in a classic example as in the following.

\section{Slender Body Motion in Wave Medium}

The problem of a body moving arbitrarily in a wave medium, which is absolutely interesting, has not been solved so completely as body in incompressible fluid. The reason for it is that the unsteady motion of a body will generate radiating waves, consequently one can not use Newton's second law to calculate the net force. Nevertheless there are good results for steady flows. Studying steady irrotational subsonic flows, [8] proved that the momentum mass is always equal to drift mass, and the kinetic energy mass is equal to the drift mass under certain conditions. [9] showed further that for some linear or even nonlinear periodic water waves and sound waves the total energy is equal to $E=m c^{2}$, where $m$ is the drift mass per wave length and $c$ is the wave velocity.

Let's now formulate the problem in a very simple way. Cartesian coordinates are set at this moment in the quiet medium and of course the absolute time is used. In the fluid potential flow theory, neglecting nonlinear and dispersive effects, the problem of an arbitrary body motion is then governed by a standard wave equation in the field,

$$
\frac{\partial^{2}}{\partial t^{2}} \varphi-c^{2} \nabla^{2} \varphi=0
$$

and the boundary condition on the rigid body surface,

$$
\frac{\partial \varphi}{\partial n}=\boldsymbol{v} \cdot \boldsymbol{n},
$$

This formulation is valid for both aerodynamic flow and shallow water flow caused by a moving body. For the aerodynamic flow, the problem is usually three-dimensional, where $\varphi$ is velocity potential, $\nabla^{2}=\frac{\partial^{2}}{\partial x^{2}}+\frac{\partial^{2}}{\partial y^{2}}+\frac{\partial^{2}}{\partial z^{2}}$, and $c$ is the sound speed. For 
the shallow water flow, it is actually two-dimensional in the horizontal plane, where $\varphi$ can be understood as a depth-averaged velocity potential, $\nabla^{2}=\frac{\partial^{2}}{\partial x^{2}}+\frac{\partial^{2}}{\partial y^{2}}$ and $c$ is the shallow water wave speed, which is equal to $\sqrt{g h}, g$ is the gravitational acceleration and $h$ the water depth. The momentum and added mass definitions in (2) and (3) are still valid for this case.

\subsection{Steady Motion of a Slender Body}

Especially we consider 2-D problem of a slender body moving along the positive $x$-axis with a constant speed $v$, i.e. steady shallow-water flow around a slender ship or steady aerodynamic flow around a 2-D thin airfoil. Cartesian coordinates are now taken to move with the body, notated by $\left(x^{\prime}, y^{\prime}\right)$. Most conveniently the body longitudinal axis is taken as the axis of $x$. Via a translation of coordinates

$$
x=x^{\prime}+v t^{\prime}, \quad y=y^{\prime}, \quad t=t^{\prime},
$$

the steady problem is then governed by

$$
\left(1-\beta^{2}\right) \varphi_{x^{\prime} x^{\prime}}+\varphi_{y^{\prime} y^{\prime}}=0,
$$

where $\beta=v / c$, a popular notation of the body-wave-speed ratio in the special relativity, is depth Froude number $\left(F_{n h}=v / \sqrt{g h}\right.$ ) for the shallow water flow or Mach number $\left(M_{\infty}=v / c_{\infty}\right)$ for the aerodynamic flow. The boundary condition on the body surface is

$$
\frac{\partial \varphi}{\partial n}=\boldsymbol{v} \cdot \boldsymbol{n}=v n_{1},
$$

where $\boldsymbol{v}=v \mathbf{i}$ and $\boldsymbol{n}=\left(n_{1}, n_{2}, n_{3}\right)$ is unit normal vector towards the outside of body.

According to the slender body theory of [2] the boundary condition for a slender body can be approximated as

$$
\frac{\partial \varphi}{\partial y^{\prime}}\left(x^{\prime}, y^{\prime}= \pm 0\right)=\mp v f_{ \pm}^{\prime}\left(x^{\prime}\right),-l / 2<x^{\prime}<l / 2,
$$

where $y^{\prime}= \pm f_{ \pm}\left(x^{\prime}\right)$ denotes the upper and lower body surfaces, $f_{ \pm}^{\prime}\left(x^{\prime}\right)=\frac{\partial}{\partial x^{\prime}} f_{ \pm}\left(x^{\prime}\right)$, and $x= \pm l / 2$ denotes two ends of the body. Without loss of generality the body can be assumed to be symmetric about the $x$-axis, i.e. $f_{+}(x)=f_{-}(x)=f(x)$. The hydrodynamic mass in (3) still holds generally for this case and its component in longitudinal direction is accordingly simplified as

$$
m=2 \rho \int_{-l / 2}^{1 / 2} \varphi \frac{\partial \varphi}{\partial y^{\prime}} \mathrm{d} x^{\prime},
$$

where $\phi$ is unit potential, i.e. $\varphi=v \phi$.

In the following we want to derive the relation of velocity dependence of hydrodynamic mass by a self-similar solution. We do a transform for the subcritical (subsonic) case $\beta<1$ as

$$
\xi=\frac{1}{\sqrt{1-\beta^{2}}} x^{\prime}
$$


Then Equation (7) becomes a standard Laplace equation,

$$
\frac{\partial^{2}}{\partial \xi^{2}} \phi+\frac{\partial^{2}}{\partial y^{\prime 2}} \phi=0
$$

with the boundary condition

$$
\begin{gathered}
\frac{\partial \phi}{\partial y^{\prime}}\left(\sqrt{1-\beta^{2}} \xi, y^{\prime}= \pm 0\right)=\mp f^{\prime}\left(\sqrt{1-\beta^{2}} \xi\right), \\
-l /\left(2 \sqrt{1-\beta^{2}}\right)<\xi<l /\left(2 \sqrt{1-\beta^{2}}\right) .
\end{gathered}
$$

On the other hand we have the Laplace equation in the case of infinitesimal velocity $\beta=0$. As we denote the variables by a subscript $o$ for this case, $\phi_{o}\left(x^{\prime}, y^{\prime}\right)$ is the solution of

$$
\frac{\partial^{2}}{\partial x^{\prime 2}} \phi_{o}+\frac{\partial^{2}}{\partial y^{\prime 2}} \phi_{o}=0
$$

with

$$
\frac{\partial \phi_{o}}{\partial y^{\prime}}\left(x^{\prime}, y^{\prime}= \pm 0\right)=\mp f^{\prime}\left(x^{\prime}\right),-l / 2<x^{\prime}<l / 2 .
$$

Now we need to compare (12) and (13) to (14) and (15). For any positive real numbers $a$ and $k$, we have a self-similar solution $\phi(a \xi, \eta)=k \phi_{o}(a \xi, a \eta)$, which satisfies

$$
\frac{\partial^{2}}{\partial \xi^{2}} \phi+\frac{\partial^{2}}{\partial \eta^{2}} \phi=k\left(\frac{\partial^{2}}{\partial \xi^{2}} \phi_{o}+\frac{\partial^{2}}{\partial \eta^{2}} \phi_{o}\right)=k a^{2}\left(\frac{\partial^{2}}{\partial x^{\prime 2}} \phi_{o}+\frac{\partial^{2}}{\partial y^{\prime 2}} \phi_{o}\right)=0
$$

with

$$
\frac{\partial \phi}{\partial \eta}(a \xi, \eta= \pm 0)=k \frac{\partial \phi_{o}}{\partial \eta}(a \xi, \eta= \pm 0)=\mp a k f^{\prime}(a \xi), \quad-l / 2<a \xi<l / 2 .
$$

Identifying (16), (17) with (12), (13) and taking $a=\sqrt{1-\beta^{2}}, k=1 / a, x^{\prime}=a \xi$ and $y^{\prime}=\eta$, we obtain the following important relation,

$$
\phi\left(x^{\prime}, y^{\prime}\right)=\frac{1}{a} \phi_{o}\left(x^{\prime}, a y^{\prime}\right) .
$$

Now it is time to carry out the result. We calculate straight forward and have

$$
\begin{aligned}
m & =\rho \int_{-\frac{l}{2}}^{\frac{l}{2}} \phi\left(x^{\prime}, y^{\prime}=0\right) f^{\prime}\left(x^{\prime}\right) \mathrm{d} x^{\prime} \\
& =\frac{\rho}{a} \int_{-\frac{l}{2}}^{\frac{l}{2}} \phi_{o}(x, y=0) f^{\prime}(x) \mathrm{d} x \\
& =\frac{m_{o}}{\sqrt{1-\beta^{2}}} .
\end{aligned}
$$

This is the velocity dependence relation of hydrodynamic mass in the slender body case, which is same as that of real mass in relativistic physics.

\subsection{Associated Medium Momentum and Energy}

According to the result of [8], the momentum caused by the steadily moving body is 
clearly expressed as

$$
p=m(\beta) v
$$

But, if the body accelerates in the medium, the total force experienced by the body is not in the form of $\mathrm{d}(\mathrm{mv}) / \mathrm{d} t$, unlike in the incompressible fluid that the total force is equal to the added mass times the acceleration, because there is extra momentum change due to wave radiation that is generated in the unsteady motion. Here the total momentum can be divided into two parts, one part $m v$ due to the steady motion at a transient moment, called carried momentum, and the other due to the radiation, called radiated momentum. These two names are proposed here tentatively. Correspondingly the total force is divided as $F=F_{c}+F_{r}$, where $F_{c}$ is due to change of carried momentum, which obeys the more general form of Newton's second law,

$$
\boldsymbol{F}_{c}=\frac{\mathrm{d}}{\mathrm{d} t} \boldsymbol{p}=\frac{\mathrm{d}}{\mathrm{d} t}(m \boldsymbol{v})=\frac{\mathrm{d} m}{\mathrm{~d} t} \boldsymbol{v}+m \frac{\mathrm{d} \boldsymbol{v}}{\mathrm{d} t},
$$

and $F_{r}$ is due to radiation. The work done by each force becomes each corresponding kinetic energy in the medium. Since the carried momentum is more compacted around the body than the radiated one, the former is physically more meaningful than the latter. Therefore only the carried kinetic energy is discussed in this paper, although the other might be able to be calculated explicitly. It is believed that the radiated kinetic energy is much smaller than the carried one in the case of either $\beta \ll 1$ or relatively small acceleration $a \ll c^{2} / L$, where $L$ is a typical length in the problem considered.

Exactly in the same way as in the derivation in relativistic dynamics, one can calculate the carried kinetic energy $T_{c}$ or the carried total energy $E_{c}$ of the medium. Begin by imagining that a force exerted $F_{c}$ on a rigid body as it moves through a distance $\mathrm{d} x$. Then its change in carried kinetic energy will be

$$
\mathrm{d} T_{c}=F_{c} \mathrm{~d} x=\frac{\mathrm{d} p}{\mathrm{~d} t} \mathrm{~d} x=v \mathrm{~d} p=v^{2} \mathrm{~d} m+m v \mathrm{~d} v .
$$

From the velocity dependence of the hydrodynamic mass (19), namely,

$$
\mathrm{d} m=\gamma^{3} m_{o} \beta \mathrm{d} \beta \text { with } \gamma=\frac{1}{\sqrt{1-\beta^{2}}},
$$

we have

$$
\mathrm{d} T_{c}=m_{o} c^{2}\left[\gamma^{3} \beta^{3} \mathrm{~d} \beta+\gamma \beta \mathrm{d} \beta\right]=\gamma^{3} m_{o} c^{2} \beta \mathrm{d} \beta,
$$

which integrates to

$$
T_{c}=m_{o} c^{2}[\gamma-1],
$$

where the constant of integration is chosen so that $T_{c}=0$ when $\beta=0$, which is identical formally to that in [4]. In the extreme limit $(\beta \rightarrow 1)$ the carried kinetic energy in this expression goes to infinity, and in the no-wave limit $(\beta \rightarrow 0)$ an expansion of $\gamma$ in (24) gives $T=m_{o} v^{2} / 2$ as well known. The term $m_{o} c^{2}$ can be seen as a kind of internal energy by virtue of the formula for wave energy $E=m_{d} c^{2}$ in [9], where $m_{d}$ is the drift mass, i.e. it is a potential of wave energy, called $U_{o}$. Thus, adding this to the 
carried kinetic energy in (24) yields a total carried energy

$$
E_{c}=T_{c}+U_{o}=m_{o} c^{2}[\gamma-1]+m_{o} c^{2}=\gamma m_{o} c^{2}=m c^{2} .
$$

\subsection{Remarks}

- It is clear that the same result as (19) holds also for 3-D slender body subsonic flows.

- The result (19) doesn't exactly hold for a non-slender body, since there is no selfsimilar solution. But it is true that the hydrodynamic mass is dependent of body moving speed and goes to infinity when $\beta$ tends to unit.

- Based on the fact that there is no loss of kinetic radiated energy in the incompressible flow, we may expect that the kinetic radiated energy due to radiation can be neglected, if $\beta$ is small or the accelerator is relatively small.

\subsection{Analogy}

It is not a mere accident to have the same mathematical forms of the mass and energy, but it is a finding of a common nature, i.e. an intrinsic analogy, in these two different contexts. This means we can extend our understanding in one context to the other one. First of all from understanding of the hydrodynamic mass, we can say the mass in physics should be a concept of field, although a compact mass point is usually regarded as confined in a finite space. It manifests as mass, the measure of the inertia, only through interacting with certain field. Indeed the physical reason for the mass increase is the interaction with its own wave medium field.

\section{Inferences}

Two dynamic properties of the special theory of relativity, as stated in the introduction, are regarded as consequences of its kinematics, i.e. consequences of Lorentz transformation. But such properties exist also in Newtonian wave mechanics. It makes one trace an analogy between the both, where the hydrodynamic mass corresponds to the real mass in relativity. It suggests that the physical reason for the velocity dependence of mass is presence of wave medium and interaction of the considered mass with the wave medium. Therefore it is actually an "effect of wave medium". In this sense the vacuum is not nothing, but material. The belief on it is not exceptional in physics community, e.g. [10] has already proposed the concept of vacuum medium.

Being similar to the special theory of relativity, it follows from (19) that the hydrodynamic mass of a moving body (its medium inertial property) is a function of its velocity. This means that a constant force exerted on a body does not result in a constant acceleration. If the radiated momentum is neglected, the relationship between force and acceleration must be found by using the general form of Newton's second law (21) which takes changing hydrodynamic mass into account. It means that a body can never be made to reach or exceed the critical speed. Dynamically, we see that the faster the body goes, the greater is the force required to accelerate it by a given amount. This is not only because of the first term in (21), but also because the mass in the second term becomes larger with increased velocity. As the body approaches the critical speed, 
its hydrodynamic mass approaches infinity. It follows that no finite force can push the body up to the critical speed.

We should keep in mind that 1) in the special theory of relativity, this dynamic explanation of the existence of a limiting velocity is derived from the fundamental kinematic limitation; 2) in the classic wave mechanics this limitation is caused by the mathematic linearization of the physic problem. We know that there are supersonic airplanes and supercritical ships in shallow water in the reality. The sound speed or the shallow water wave speed is really a barrier for airplanes or ships, but never a limit. One will naturally ask whether the speed of light is the limiting velocity in our universe. A philosophic answer is of course "No". Then how can we imagine what happens if an object moves through the transluminal speed range and finally at a superluminal speed? Let's first ignore the transition scene from the sub-luminal to super-luminal speed and just give a qualitative answer to the superluminal one.

Assume this analogy traced in this paper is true also for the supercritical case. This means we are permitted to use one result in hydrodynamics to explain phenomenologically the other in electrodynamics or inversely. As well-known in Newtonian wave mechanics, a body moving at a supercritical speed (e.g. a plane at a supersonic speed), will generate waves (oblique shock waves), which radiate to infinity. Due to the loss of momentum of radiated waves, the body will experience a resistance, even if the body moves at a constant speed. Similar phenomenon happens in Cherenkov radiation, c.f. [11] (p. 638) and [12]. This means that

- in the supercritical range one can never find a reference frame with constant speed that is an inertial frame;

- no object can move freely (without any external supporting force in an infinite space) at a supercritical speed!

This scene and its consequences are applicable to the superluminal case.

There are nevertheless exceptional examples of free motions at supercritical speeds both in Newtonian wave mechanics and electromagnetism. It was found theoretically and experimentally in [13] and [14] that a slender body can move freely without experiencing any wave resistance at a certain supercritical speed in a narrow shallow water channel. This can be explained by both linear and nonlinear shallow water wave theories. This idea can be extended to electrodynamics. Two massless particles with equal charge but different signs (positive and negative) are connected firmly to each other by a massless rod. Suppose this particle system moves at a superluminal speed along the central line of a tube, of which the wall can reflect the electromagnetic wave perfectly. The particle at the front (with the positive charge) will generate a conic wave and this wave will be reflected by the wall and reach the particle at the back (with the negative charge). This reflected wave can be absorbed by the particle at the back totally under certain kinematic condition, as shown in Figure 1. This means there is no wave in the wake, or no wave outward radiated, therefore there is no wave resistance experienced by the particle combination system.

There is another superluminal example in the electromagnetism, which might be explained qualitatively by this analogy. [15] discovered experimentally that electromagnetic 


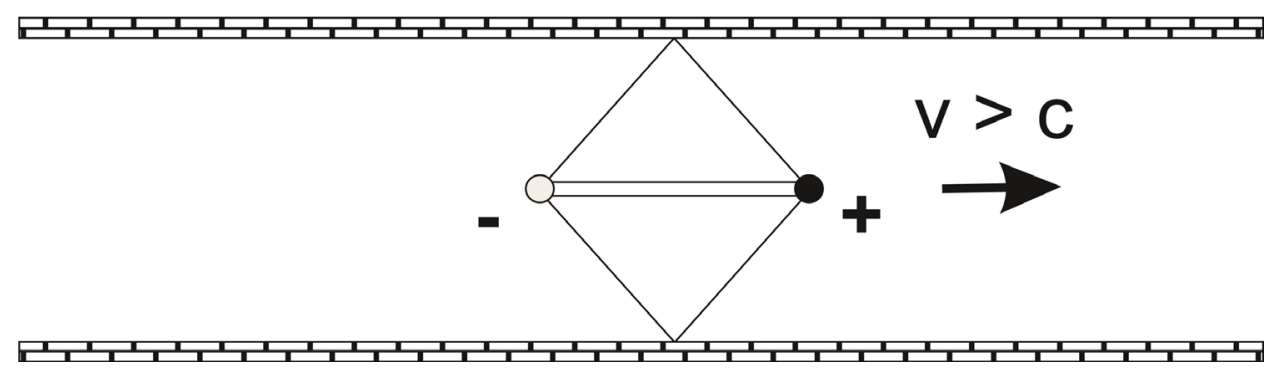

Figure 1. Inferred (proposed) superluminal electromagnetic wave structure that is generated by a combination of two massless particles with equal charge but different signs moving at a superluminal speed in a tunnel.

microwave groups can go through a tunnel at a superluminal speed. Because of the presence of side wall of tunnel, the waves will form a rhombic pattern through side wall reflection, of which the phase velocity is already large than the light speed. However in the linear wave theory, the group velocity of this wave pattern in the longitudinal direction is always less than the light speed. This means the linear theory cannot explain this phenomenon. Nevertheless there are particular examples in nonlinear shallow water waves to show the drift mass (energy) propagation velocity is larger than the critical speed. One is the oblique interaction of two solitary waves (the cross wave system), e.g. as shown in Fig. 8 on p. 1588 and Fig. 9 on p. 1589 in [16]. The cross point will move at a supercritical speed and bring a drift mass with it, i.e. the drift mass (energy) propagates at a supercritical speed, which is significantly larger than the critical speed. The other example of the so-called Mach reflexion, is similar to the former one and more clear for understanding. It was shown theoretically and experi- mentally e.g. in [17] and [18] that for a solitary wave incident obliquely upon a straight wall, if the angle of incidence is small, additionally to the incident and reflected waves there is a third wave crest called Mach stem, which intersects the wall normally and propagates along the wall at a supercritical speed.

\section{Conclusion}

The slender body motion in the classical wave media possesses the same dynamic properties of two famous Equations (19) and (25) as in the special theory of relativity. But they are derived here purely classically, namely, in terms of the absolute coordinate system, rather than in terms of the relative space-time. Main inferences drawn from this analogy are that the increase of mass with velocity is due to the interaction of moving body (particle) with wave medium field and the vacuum is the medium that supports electromagnetic waves. A further thinking result is that no object can move freely in an unbounded space at a constant superluminal speed, but it can do in a channel (tunnel)!

\section{Acknowledgements}

The author would like to thank Professor S. D. Sharma in retirement at the Mercator University of Duisburg for his valuable discussions and comments, especially for pointing out Lagally's theorem, given e.g. in [19], which is the starting point for the comparison in Appendix, and the paper on the discovery of the superluminal phenomenon [15]. 


\section{References}

[1] Michell, J.H. (1898) The Wave-Resistance of a Ship. Philosophical Magazine, 45, 106-123. http://dx.doi.org/10.1080/14786449808621111

[2] Tuck, E.O. (1966) Shallow Water Flows Past Slender Bodies. Journal of Fluid Mechanics, 26, 81-95. http://dx.doi.org/10.1017/S0022112066001101

[3] Einstein, A. (1905) Zur Elektrodynamik bewegter Körper. Annalen der Physik, 17, 891-921. http://dx.doi.org/10.1002/andp.19053221004

[4] Einstein, A. (1905) Ist die Trägheit eines Körpers von seinem Energieinhalt abhängig? Annalen der Physik, 18, 639-641. http://dx.doi.org/10.1002/andp.19053231314

[5] Milne-Thomson, L.M. (1968) Theoretical Hydrodynamics. 5th Edition, Dover Publications, New York. http://dx.doi.org/10.1007/978-1-349-00517-8

[6] Pais, A. (1982) "Subtle Is the Lord..." the Science and the Life of Albert Einstein. Oxford Univ. Press, Oxford.

[7] Thomson, J.J. (1881) On the Electric and Magnetic Effects Produced by the Motion of Electrified Bodies. Philosophical Magazine, 11, 229-249. http://dx.doi.org/10.1080/14786448108627008

[8] Yih, C.-S. (1995) Kinetic-Energy Mass, Momentum Mass, and Drift Mass in Steady Irrotational Subsonic Flows. Journal of Fluid Mechanics, 297, 29-36. http://dx.doi.org/10.1017/S0022112095002989

[9] Yih, C.-S. (1997) The Role of Drift mass in the Kinetic Energy and Momentum of Periodic Water Waves and Sound Waves. Journal of Fluid Mechanics, 331, 429-438. http://dx.doi.org/10.1017/S0022112096003539

[10] Shupe, M.A. (1985) The Lorentz-Invariant Vacuum Medium. American Journal of Physics, 53, 122-127. http://dx.doi.org/10.1119/1.14094

[11] Jackson, J.D. (1975) Classical Electrodynamics. 2nd Edition, John Wiley \& Sons, New York.

[12] Carusotto, I. and Rousseaux, G. (2013) The Cerenkov Effect Revisited: From Swimming Ducks to Zero Modes in Gravitational Analogues. In: Analogue Gravity Phenomenology. Springer International Publishing, Berlin, 109-144.

[13] Chen, X.-N. and Sharma, S.D. (1997) Zero Wave Resistance for Ships Moving in Shallow Channels at Supercritical Speeds. Journal of Fluid Mechanics, 335, 305-321. http://dx.doi.org/10.1017/S0022112096004533

[14] Chen, X.-N., Sharma, S.D. and Stuntz, N. (2003) Zero Wave Resistance for Ships Moving in Shallow Channels at Supercritical Speeds. Part 2. Improved Theory and Model Experiment. Journal of Fluid Mechanics, 478, 111-124. http://dx.doi.org/10.1017/S0022112002003178

[15] Enders, A. and Nimtz, G. (1992) On Superluminal Barrier Traversal. J. Phys. I, 2, 16931698. http://dx.doi.org/10.1051/jp1:1992236

[16] Soomere, T. (2009) Solitons Interactions. In: Meyers, R.A., Ed., Encyclopedia of Complexity and Systems Science, Springer, New York, 8479-8504.

http://dx.doi.org/10.1007/978-0-387-30440-3_507

[17] Miles, J.W. (1977) Resonantly Interacting Solitary Waves. Journal of Fluid Mechanics, 79, 171-179. http://dx.doi.org/10.1017/S0022112077000093

[18] Melville, W.K. (1980) On the Mach Reflection of Solitary Waves. Journal of Fluid Mechanics, 98, 285-297. http://dx.doi.org/10.1017/S0022112080000158

[19] Betz, A. (1932) Singularitätenverfahren zur Ermittlung der Kräfte und Momente auf Körper in Potentialströmungen. Achieve of Applied Mechanics, 3, 454-462.

http://dx.doi.org/10.1007/bf02079821 


\section{Appendix: Comparison of Electrodynamics and Hydrodynamics}

Electrodynamics has different fundamentals from hydrodynamics. But the three basic laws of Coulomb, Ampère and Faraday have same or similar mathematical forms as or to some theorems or laws in hydrodynamics. Here some known analogies or possibly unknown analogies between electrodynamics and hydrodynamics are parallelly listed. The electrodynamic laws are referred to [11] and hydrodynamic laws and theorems to [5] and [19].

\section{Electrodynamics}

Coulomb's Law

$$
\mathbf{F}=q \mathbf{E} \Longleftrightarrow \nabla \cdot \mathbf{E}=4 \pi \rho
$$

Ampère's Law

$$
\mathbf{F}=\mathbf{J} \times \mathbf{B} \Longleftrightarrow \nabla \times \mathbf{B}=(4 \pi / c) \mathbf{J}
$$

\section{Hydrodynamics}

Lagally's Theorem

$$
\mathbf{F}=Q \mathbf{v} \Longleftrightarrow \nabla \cdot(\rho \mathbf{v})=Q
$$

Kutta and Joukowsky Theorem

$$
\mathbf{F}=\rho \mathbf{v} \times \omega \Longleftrightarrow \nabla \times \mathbf{v}=\omega
$$

1)

Biot and Savart Law (or Theorem)

$$
F_{12}=\begin{gathered}
I_{1} I_{2} \\
\omega_{1} \omega_{2}
\end{gathered} \oint \oint \frac{d \mathbf{l}_{\mathbf{1}} \times\left(d \mathbf{l}_{\mathbf{2}} \times \mathbf{x}_{\mathbf{1 2}}\right)}{\left|x_{12}\right|^{3}}
$$

Faraday's Law of Induction

$$
\begin{gathered}
-\frac{1}{c} \frac{d}{d t} \int_{S} \mathbf{B} \cdot \mathbf{n} d S=\oint_{C} \mathbf{E} \cdot d \mathbf{l} \\
\Longleftrightarrow \nabla \times \mathbf{E}+\frac{1}{c} \frac{\partial \mathbf{B}}{\partial t}=0
\end{gathered}
$$

Charge Conservation

$$
\frac{\partial \rho}{\partial t}+\nabla \cdot \mathbf{J}=0
$$

Lorentz Gauge with vector and scalar potentials

$$
\nabla \cdot \mathbf{A}+\frac{1}{c} \frac{\partial \Phi}{\partial t}=0
$$

Maxwell Equations

$$
\begin{gathered}
\nabla \cdot \mathbf{E}=4 \pi \rho \quad \nabla \times \mathbf{B}=\frac{4 \pi}{c} \mathbf{J}+\frac{1}{c} \frac{\partial \mathbf{E}}{\partial t} \\
\nabla \cdot \mathbf{B}=0 \quad \nabla \times \mathbf{E}+\frac{1}{c} \frac{\partial \mathbf{B}}{\partial t}=0 \\
\Longleftrightarrow\left(\frac{\partial^{2}}{\partial t^{2}}-c^{2} \nabla^{2}\right)\left\{\begin{array}{c}
\Phi \\
\mathbf{A}
\end{array}\right\}=0
\end{gathered}
$$

Newton's Second Law or Bernoulli's Theorem

$$
\begin{gathered}
\frac{d}{d t} \int_{V} \rho \mathbf{v} d V=-\int_{S} p \mathbf{n} \cdot d S \\
\Longleftrightarrow \frac{\partial(\rho \mathbf{v})}{\partial t}+\nabla p=0 \quad \text { (linearised) }
\end{gathered}
$$

Mass Conservation

$$
\frac{\partial \rho}{\partial t}+\nabla \cdot(\rho \mathbf{v})=0
$$

Material State Equation, e.g.

$$
p \sim \rho^{\gamma}
$$

Wave Equations

$$
\left(\frac{\partial^{2}}{\partial t^{2}}-c^{2} \nabla^{2}\right)\left\{\begin{array}{l}
\rho \\
p \\
\mathbf{v}
\end{array}\right\}=0
$$

Some remarks concerning what we can learn from the comparison are given here.

- Both the electric field $\boldsymbol{E}$ and magnetic field $\boldsymbol{B}$ correspond to the velocity field $v$ or the momentum field $\rho \boldsymbol{v}$.

- The gauge in the electromagnetism is quite strange to a continuum mechanist. This is actually a typical problem for the theory based on field observation, see a further explanation below.

- Assume in the fluid dynamics we would not know what is vortex, but we know it is 
a rotation of some field, which is proposed to be $\boldsymbol{B}$. Finally we find the equation system is not complete and we need a relationship between $\boldsymbol{B}$ and $\boldsymbol{v}$. This is actually the gauge here, like the relationship between $\boldsymbol{B}$ and $\boldsymbol{E}$ in the electromagnetism. In the fluid dynamics it is so simple and trivial, i.e. $\boldsymbol{B}=\boldsymbol{v}$, that one could even ignore it.

- The wave equation in the fluid dynamics could be set up based on pure field observations like the set up of Maxwell equations. It is obvious that the nonlinear convective term is missing in such a derivation. The critical wave speed becomes a singularity in the artificial mathematical problem. But this should not be a physical limitation of the body moving speed!

Submit or recommend next manuscript to SCIRP and we will provide best service for you:

Accepting pre-submission inquiries through Email, Facebook, LinkedIn, Twitter, etc. A wide selection of journals (inclusive of 9 subjects, more than 200 journals)

Providing 24-hour high-quality service

User-friendly online submission system

Fair and swift peer-review system

Efficient typesetting and proofreading procedure

Display of the result of downloads and visits, as well as the number of cited articles

Maximum dissemination of your research work

Submit your manuscript at: http://papersubmission.scirp.org/

Or contact wjm@scirp.org 